\title{
Stability Analysis and Bifurcation Control of Hysteresis Current Controlled Ćuk Converter Using Filippov's Method
}

\section{Daho*, D. Giaouris*(Member IET), B. Zahawi*(Member IET), V. Picker*(Member IET) and S. Banerjee**}

*School of Electrical, Electronic and Computer Engineering, University of Newcastle upon Tyne,UK. Ibrahim.daho@ncl.ac.uk ** Department of Electrical Engineering, Indian Institute of Technology, Kharagpur, India. soumitro@ee.iitkgp.ernet.in

Keywords: Ćuk converter, Bifurcation, Hysteresis controller, Filippov method.

\begin{abstract}
An autonomous Ćuk converter controlled by a hysteresis current controller is studied in this paper. Filippov's method is employed for the first time to investigate the stability of an autonomous power converter. The Neimark bifurcation, via which the converter loses its stabile period one operation, was stabilized by applying two alternative control strategies based on the analysis of the behaviour of the complex eigenvalues of the monodromy matrix of the system.
\end{abstract}

\section{Introduction}

The Ćuk converter with hysteresis current control is an autonomous dynamical system as it is not switched at any specific clock frequency. To date, the number of studies carried out to identify the bifurcation and chaotic behaviour patterns of autonomous converters has been very limited. Conventionally, the stable operating range of the converter is assessed using the complex eigenvalues of the system's Jacobian at the equilibrium points [1]. This technique can successfully determine the stability of the limit cycle; however, it offers little knowledge of how and why this loss of stability occurs. In this paper, Filippov's method is employed for the first time to investigate the stability of an autonomous power converter providing an insight into the nature of the problem, showing that the converter looses stability via a Neimark Bifurcation, thus allowing new control strategies to be developed to extend the range of stable period-one operation. The method has been previously applied to non-autonomous systems including the buck and the boost converters $[2,3]$.

\section{The Ćuk converter}

The Ćuk converter (Fig.1) is controlled by a hysteresis current controller, where the reference current $i_{\text {ref }}$ is compared with the sum of the inductor currents $i_{1}+i_{2}$. The switch $S$ is turned $\mathbf{O N}$ when the sum of the inductor currents $i_{1}+i_{2}$ is lower than $i_{\text {ref }}+\Delta i / 2$ and OFF when it goes above $i_{\text {ref }}-\Delta i / 2$, where $\Delta i$ is the width of the hysteretic band. The reference current $i_{\text {ref }}$ is related to the output voltage $v_{1}$. If $\Delta i$ is sufficiently small, we can write:

$i_{1}+i_{2}=k-\mu v_{1}$

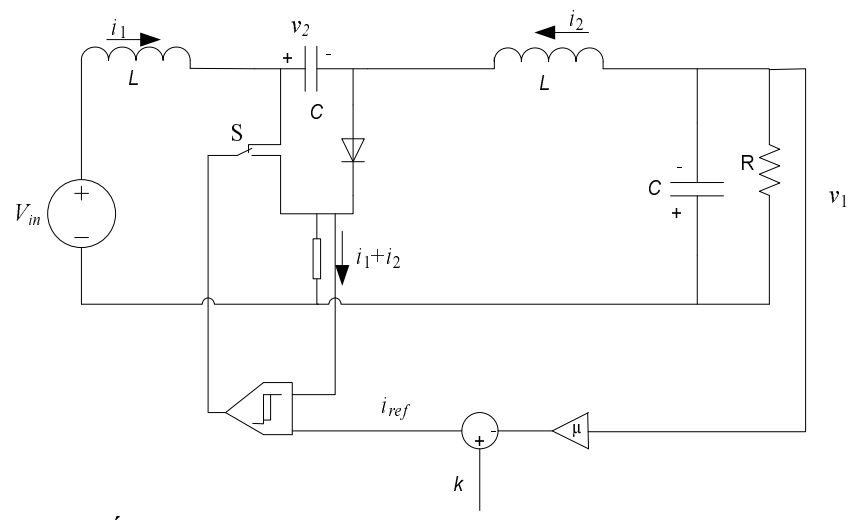

Fig. 1: Ćuk converter under hysteretic controller.

where $k$ and $\mu$ are control parameters. The converter itself is governed by two sets of linear differential equations related to the $\mathbf{O N}$ and $\mathbf{O F F}$ states of the converter. The state equations of the system can be written as:

When the switch is turned $\mathbf{O N}$ :

$\begin{cases}\dot{x}_{1}=\frac{x_{3}}{C}-\frac{x_{1}}{C R}, & \dot{x}_{2}=-\frac{x_{3}}{C} \\ \dot{x}_{3}=\frac{x_{2}}{L}-\frac{x_{1}}{L}, & \dot{x}_{4}=\frac{V_{i n}}{L}\end{cases}$

When the switch is turned OFF:

$\left\{\begin{array}{l}\dot{x}_{1}=\frac{x_{3}}{C}-\frac{x_{1}}{C R}, \dot{x}_{2}=\frac{x_{4}}{C} \\ \dot{x}_{3}=-\frac{x_{1}}{L}, \quad \dot{x}_{4}=-\frac{x_{2}}{L}+\frac{V_{i n}}{L}\end{array}\right.$

The output voltages $v_{1}=x_{1}, v_{2}=x_{2}$, and the inductor currents $i_{1}=x_{3}, i_{2}=x_{4}$ are taken as the state variables of the system. In matrix form, we can write:

$\dot{\mathbf{X}}=\mathbf{A}_{1} \mathbf{X}+\mathbf{B} u$ for $\mathbf{O N}$ state

$\dot{\mathbf{X}}=\mathbf{A}_{2} \mathbf{X}+\mathbf{B} u$ for $\mathbf{O F F}$ state

where

$\mathbf{A}_{1}=\left[\begin{array}{cccc}-1 / C R & 0 & 1 / C & 0 \\ 0 & 0 & -1 / C & 0 \\ -1 / L & 1 / L & 0 & 0 \\ 0 & 0 & 0 & 0\end{array}\right]$, 
$\mathbf{A}_{2}=\left[\begin{array}{cccc}-1 / C R & 0 & 1 / C & 0 \\ 0 & 0 & -1 / C & 0 \\ -1 / L & 0 & 0 & 0 \\ 0 & -1 / L & 0 & 0\end{array}\right], \mathbf{B} u=\left[\begin{array}{c}0 \\ 0 \\ 0 \\ V \text { in } \\ \hline L\end{array}\right]$

$\mathbf{X}=\left[\begin{array}{llll}v_{1} & v_{2} & i_{2} & i_{1}\end{array}\right]^{\mathrm{T}}$ and $u$ is the input voltage $V_{i n}$.

Figs. 2 and 3 show inductor current waveforms for two different values of the control parameter $k$. Fig. 2 shows a stable operating condition $(k=1.125)$ while a slow scale instability occurs when $k=4.125$, as shown in Fig. $3\left(L_{l}=\right.$ $L_{2}=1 \mathrm{mH} C=47 \mu \mathrm{F}, \mathrm{R}=75 \Omega, V_{\text {in }}=15 \mathrm{~V}$ and $\left.\mu=0.025\right)$.

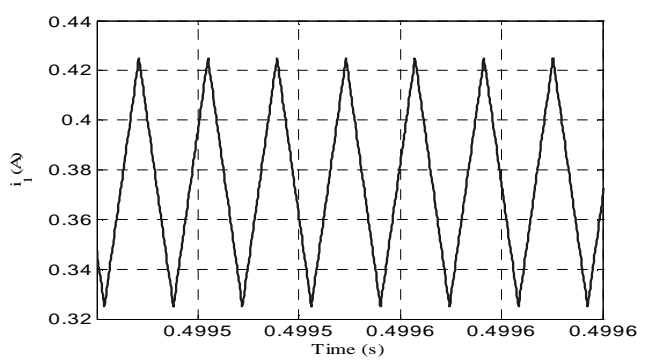

Fig. 2: Stable inductor current waveform $i_{1}$

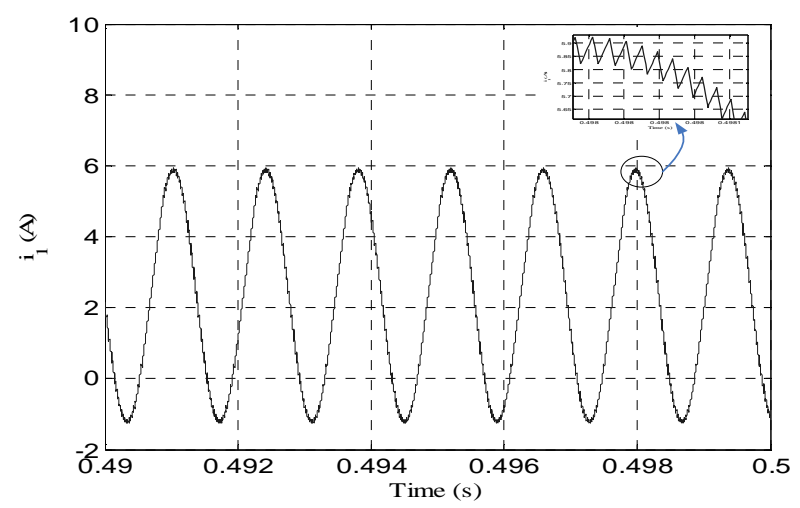

Fig. 3: Unstable inductor current waveform $i_{1}$

\section{Stability analysis using Filippov's method}

The new method is based on the Floquet multipliers (the eigenvalues of the monodromy matrix) of the system's monodromy matrix $\mathbf{W}$ : i.e. the fundamental solution matrix over one full cycle. If the system is piecewise smooth (as is the case for the hysteresis current controlled Ćuk converter), the monodromy matrix may be broken into areas where the vector field is smooth and areas where the vector field crosses a switching manifold. Suppose a periodic orbit starts at $t_{0}$ and is passing from the subsystem- 1 given by the vector field $\mathbf{f}$. $(\mathbf{x},(t))$, intersects the switching manifold defined by the algebraic equation $h(\mathbf{x}(t), t)=0$ at $t_{\Sigma}$, and goes to subsystem-2 given by the vector field $\mathbf{f}_{+}(\mathbf{x}(t))$. It has been shown $[4,5]$ that when there is a transversal intersection, the state transition matrix across the switching manifold, called also the saltation matrix $\mathbf{S}$, is given by:

$$
\mathbf{S}=\mathbf{I}+\frac{\left(\lim _{t \rightarrow t_{\Sigma+}} \mathbf{f}_{+}(\mathbf{x}(t))-\lim _{t \rightarrow t_{\Sigma-}} \mathbf{f}_{-}(\mathbf{x}(t))\right) \mathbf{h}^{T}}{\mathbf{n}^{T} \lim _{t \rightarrow t_{\Sigma-}} \mathbf{f}_{-}(\mathbf{x}(t))+\left.\frac{d h}{d t}\right|_{t=t_{\Sigma}}}
$$

Where $t_{\Sigma+}$ and $t_{\Sigma-}$ indicate the time instant just before and after the switching manifold, $t_{\Sigma}$ is the time at which the orbit crosses the switching manifold and $\mathbf{n}$ is a vector normal to the switching surface.

Then at one switching point the monodromy matrix can be composed as:

$$
\begin{aligned}
\mathbf{W}\left(t_{0}+T, t_{0},\right. & \left.x\left(t_{0}\right)\right)= \\
& \mathbf{\Phi}_{2}\left(t_{0}+T, t_{\Sigma}, x\left(t_{\Sigma}\right)\right) \times \mathbf{S} \times \mathbf{\Phi}_{1}\left(t_{\Sigma}, t_{0}, x\left(t_{0}\right)\right)
\end{aligned}
$$

$\boldsymbol{\Phi}_{1}\left(t_{\Sigma}, t_{0}, x\left(t_{0}\right)\right)$ is the state transition matrix during the first time interval (i.e. when the switch is $\mathbf{O N}), \mathbf{\Phi}_{2}\left(t_{0}+T, t_{\Sigma}, x\left(t_{\Sigma}\right)\right)$ is the state transition matrix for the second interval (i.e. when the switch is open) and $T$ is the switching period.

\section{Derivation of the monodromy matrix of the Cuk converter}

The hysteresis current controlled Ćuk converter has two switching manifolds: one where the switch is turned OFF and the other when the switch is turned $\mathbf{O N}$, as shown in Fig. 4. In order to derive the monodromy matrix of the system we need to know the two saltation matrices at the two switching manifolds. To study the stability of the period-1 orbit, it suffices to consider $t$ varying from 0 to $T$.

The switching hypersurface $h_{1}$ when the switch is turned OFF is given by:

$h_{1}(\mathbf{X},(d T))=x_{3}(d T)+x_{4}(d T)-k+\mu x_{1}-\frac{\Delta i}{2}$

The normal vector $\mathbf{n}$ is given by:

$$
\mathbf{n}^{T}=\nabla h_{1}(\mathbf{X}, d T)=\left[\begin{array}{llll}
\mu & 0 & 1 & 1
\end{array}\right]
$$

The two vector fields before and after switching OFF are:

$$
\mathbf{f}_{-}(x)=\left[\begin{array}{c}
-x_{1} / R C+x_{2} / C \\
x_{3} / C \\
-x_{1} / L+x_{2} / C \\
V_{i n} / L
\end{array}\right]_{t=d T} \quad \mathbf{f}_{+}(x)=\left[\begin{array}{c}
-x_{1} / R C+x_{2} / C \\
x_{4} / C \\
-x_{1} / L \\
-x_{2} / L+V_{i n} / L
\end{array}\right]_{t=d T}
$$

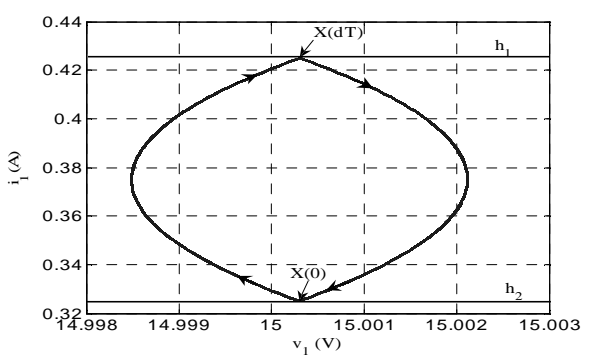

Fig. 4: Phase space and the transversal intersection.

And the saltation matrix at switching OFF is:

$$
\mathbf{S}_{\mathbf{1}}(d T)=\mathbf{I}+\frac{\left(\mathbf{f}_{+}-\mathbf{f}_{-}\right) \mathbf{n}^{T}}{\mathbf{n}^{T} \mathbf{f}_{-}}
$$


The switching hypersurface $\left(h_{2}\right)$ when the switch is turned From (16)

$\mathbf{O N}$ is given by:

$h_{2}(\mathbf{X},(T))=x_{3}(T)+x_{4}(T)-i k+\mu x_{1}+\frac{\Delta i}{2}$

The normal vector $\mathbf{n}$ is given by:

$$
\mathbf{n}^{T}=\Delta h_{2}(\mathbf{X}, T)=\left[\begin{array}{llll}
\mu & 0 & 1 & 1
\end{array}\right]
$$

The two vector fields before and after switching $\mathbf{O N}$ are:

$\mathbf{f}_{-}(x)=\left[\begin{array}{c}-x_{1} / R C+x_{2} / C \\ x_{4} / C \\ -x_{1} / L \\ -x_{2} / L+V_{i n} / L\end{array}\right]_{t=T} \quad \mathbf{f}_{+}(x)=\left[\begin{array}{c}-x_{1} / R C+x_{2} / C \\ x_{3} / C \\ -x_{1} / L+x_{2} / C \\ V_{i n} / L\end{array}\right]_{t=T}$

The saltation matrix at switching $\mathbf{O N}$ is:

$\mathbf{S}_{2}(T)=\mathbf{I}+\frac{\left(\mathbf{f}_{+}-\mathbf{f}_{-}\right) \mathbf{n}^{T}}{\mathbf{n}^{T} \mathbf{f}_{-}}$

The monodromy matrix of the system is then given by:

$\mathbf{W}(T, 0)=\mathbf{S}_{\mathbf{2}} \times \boldsymbol{\Phi}_{\mathbf{2}}(T, d T) \times \mathbf{S}_{\mathbf{1}} \times \boldsymbol{\Phi}_{\mathbf{1}}(d T, 0)$

The state transition matrix during the first time interval (i.e. when the switch is $\mathbf{O N})$ is given by $\mathbf{\Phi}_{1}(d T, 0)=e^{\mathbf{A}}{ }_{1} d t$. The state transition matrix for the second interval (i.e. when the switch is $\mathbf{O F F})$ is given by $\mathbf{\Phi}_{\mathbf{2}}(T, d T)=e^{\mathbf{A}}{ }_{2} T(1-d)$.

Hence, the Monodromy matrix becomes:

$$
\mathbf{W}(T, 0)=\mathbf{S}_{2} \times e^{\mathbf{A}_{2} T(1-d)} \times \mathbf{S}_{1} \times e^{\mathbf{A}_{1} d T}
$$

Calculation of switching period $T$

In order to calculate the transition matrices we need to know the duty cycle $d$ and the switching period T. The duty cycle $d$ can be calculated from the average model of the Ćuk converter. The switching period $T$ can be calculated as follows:

Let the duty cycle $d=t / T$ and $d^{\prime}=(1-d) T$. The value of the state victor at $t=d T$ is:

$\mathbf{X}(d T)=\boldsymbol{\Phi}_{1}(d T) \mathbf{X}(0)+\int_{0}^{d T} e^{\mathbf{A}_{1}(d T-\tau)} \mathbf{B} d \tau$

The state victor at $t=T$ is:

$$
\mathbf{X}(T)=\mathbf{X}(0)=\boldsymbol{\Phi}_{2}\left(d^{\prime} T\right) \mathbf{X}(d T)+\int_{d T}^{T} e^{\mathbf{A}_{2}(T-\tau)} \mathbf{B} d \tau
$$

Substituting (16) into (17)

$$
\begin{aligned}
\mathbf{X}(0) & =\left[\mathbf{I}-\boldsymbol{\Phi}_{2}\left(d^{\prime} T\right) \boldsymbol{\Phi}_{\mathbf{1}}(d T)\right]^{-1} \times \boldsymbol{\Phi}_{2}\left(d^{\prime} T\right) \\
& \times \int_{0}^{d T} e^{\mathbf{A}_{1}(d T-\tau)} \mathbf{B} d \tau+\int_{d T}^{T} e^{\mathbf{A}_{2}(T-\tau)} \mathbf{B} d \tau
\end{aligned}
$$

From the hypersurface at the switching OFF point

$$
x_{3}(d T)+x_{4}(d T)=k-\mu x_{1}+\frac{\Delta i}{2}
$$

$$
\begin{aligned}
x_{3}(d T)+x_{4}(d T)= & {\left[\begin{array}{llll}
0 & 0 & 1 & 1
\end{array}\right] } \\
& \times\left(\boldsymbol{\Phi}_{\mathbf{1}}(d T) \mathbf{X}(0)+\int_{0}^{d T} e^{\mathbf{A}_{1}(d T-\tau)} \mathbf{B} d \tau\right)
\end{aligned}
$$

Hence

$$
\begin{aligned}
& {\left[\begin{array}{llll}
0 & 0 & 1 & 1
\end{array}\right] \times\left(\boldsymbol{\Phi}_{\mathbf{1}}(d T) \mathbf{X}(0)+\int_{0}^{d T} e^{\mathbf{A}_{\mathbf{1}}(d T-\tau)} \mathbf{B} d \tau\right)} \\
& -\left(k-\mu x_{1}+\frac{\Delta i}{2}\right)=0
\end{aligned}
$$

Substituting $\boldsymbol{X}$ (0) from (18) we can solve (21) using the simulating annealing (SA) method to obtain the switching period $T$. Knowing $d$ and $T$, it is thus possible to calculate the state vector $\boldsymbol{X}(d T), \boldsymbol{X}(0)$, the two saltation matrices $\mathbf{S}_{1}$ and $\mathbf{S}_{2}$, and hence the monodromy matrix of the system (14).

\section{Eigenvalues of the monodromy matrix}

Using the monodromy matrix, we can investigate the possible bifurcation behavior exhibited by the period-1 orbits. The stability analysis can be obtained by examining the evaluation of the eigenvalues of the monodromy matrix, as parameters are varied. If all the eigenvalues of the monodromy matrix are inside the unit circle, the system is stable. Any crossing of the eigenvalues out of the unit circle indicates a loss of stability.

For $V_{\text {in }}=15 \mathrm{~V}, L=1 \mathrm{mH}, \mathrm{C}=47 \mu \mathrm{F}, R=40 \Omega, \mu=0.025, k=1.125$ and $d=0.5$. The SA solution of equation (21) gives $T=13.655$ $\mu \mathrm{s}$. The state vectors when switching $\mathbf{O F F}, \boldsymbol{X}(d T)$, and when switching ON, $\boldsymbol{X}(0)$ can now be calculated from (16) and (17):

$$
\begin{aligned}
\mathbf{X}(d T) & =\left[\begin{array}{llll}
14.9887 & 29.9487 & 0.4253 & 0.4252
\end{array}\right]^{T} \\
\mathbf{X}(0) & =\left[\begin{array}{llll}
14.9882 & 30.0024 & 0.325 & 0.3247
\end{array}\right]^{T}
\end{aligned}
$$

And the saltation matrices $S_{1}$ and $S_{2}$ :

$$
\mathbf{S}_{1}=\left[\begin{array}{cccc}
1 & 0 & 0 & 0 \\
0.0154 & 1 & 0.6171 & 0.6171 \\
-0.025 & 0 & 0.0013 & -0.9987 \\
-0.0250 & 0 & -0.9987 & 0.0013
\end{array}\right]
$$

$$
\mathbf{S}_{\mathbf{2}}=\left[\begin{array}{cccc}
1 & 0 & 0 & 0 \\
0.0118 & 1 & 0.4700 & 0.4700 \\
-0.025 & 0 & 0.0005 & -0.9995 \\
-0.0250 & 0 & -0.9995 & 0.0005
\end{array}\right]
$$

The monodromy matrix is thus given by:

$$
\mathbf{W}(0, X(0), T)=\left[\begin{array}{cccc}
0.9881 & 0.0005 & 0.1454 & -0.1462 \\
-0.0027 & 0.9958 & -0.1468 & 0.1447 \\
-0.0065 & 0.0135 & 1 & 0.0060 \\
0.0068 & 0 & -0.0008 & 1
\end{array}\right]
$$


The eigenvalues of the monodromy are:

$$
\operatorname{eig}(\mathbf{W}(0, X(0), T))=\left[\begin{array}{c}
1 \\
0.9927 \\
0.9963 \pm 0.0618
\end{array}\right]
$$

As all the eigenvalues are inside the unit circle, the system for the above parameters is stable as expected and shown in Fig. 2. We will now examine the stability of the system as one of the control parameters $k$ is changed keeping $\mu$ constant. Table 1 shows the Floquet multipliers of the monodromy matrix as $k$ is varied. The monodromy matrix has two real eigenvalues and a complex conjugate pair. As the system is an autonomous system, one of the real eigenvalues is always 1 and the other is close to unity. Also, they do not significantly change with the changes in the bifurcation variable; therefore, the two real eigenvalues do not influence the stability of the system. As $k$ increases the complex eigenvalues move out of the unit circle. A Neimark bifurcation occurs just before $k=4.125$, and the system loses stability as expected (shown in Fig. 3). Fig. 5 shows the locations of the eigenvalues as $k$ is varied from 1.125 to 4.125 .

\begin{tabular}{c|c|c|c|c}
\hline \multirow{2}{*}{$K$} & \multicolumn{3}{|c|}{} & $\begin{array}{l}\text { Absolute } \\
\text { value of the } \\
\text { complex pair }\end{array}$ \\
\hline 1.125 & $0.9963 \pm 0.0618 \mathrm{i}$ & 1 & 0.9927 & 0.9982 \\
\hline 1.5 & $0.9970 \pm 0.0561 \mathrm{i}$ & 1 & 0.9933 & 0.9986 \\
\hline 1.875 & $0.9976 \pm 0.0519 \mathrm{i}$ & 1 & 0.9938 & 0.9989 \\
\hline 2.25 & $0.9979 \pm 0.0495 \mathrm{i}$ & 1 & 0.9941 & 0.9992 \\
\hline 2.625 & $0.9983 \pm 0.0467 \mathrm{i}$ & 1 & 0.9944 & 0.9994 \\
\hline 3 & $0.9985 \pm 0.0470 \mathrm{i}$ & 1 & 0.9944 & 0.9996 \\
\hline 3.375 & $0.9987 \pm 0.0458 \mathrm{i}$ & 1 & 0.9945 & 0.9998 \\
\hline 3.75 & $0.9991 \pm 0.0419 \mathrm{i}$ & 1 & 0.9945 & 0.9999 \\
\hline $\mathbf{4 . 1 2 5}$ & $\mathbf{0 . 9 9 9 1 \pm 0 . 0 4 4 i}$ & $\mathbf{1}$ & $\mathbf{0 . 9 8 7 1}$ & $\mathbf{1 . 0 0 0 1}$ \\
\hline
\end{tabular}

Table 1: Eigenvalues of the monodromy matrix as $k$ is varied.

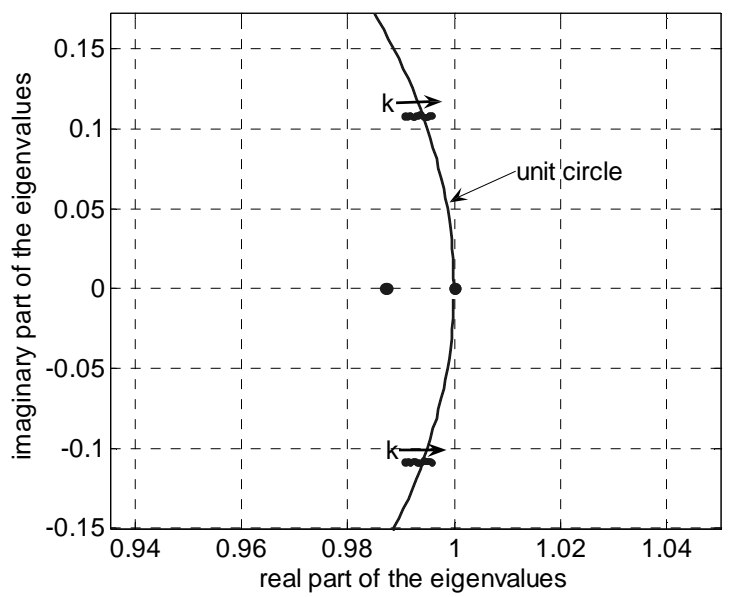

Fig. 5: Closed-up view of the locus of the eigenvalues.

\section{Controlling the Neimark Bifurcation}

Previously, the system has been studied [1] using the complex eigenvalues of the system's Jacobian at the equilibrium points. This technique can successfully determine the stability of the limit cycle. However, it offers little knowledge of how this loss of stability occurs. The use of Filippov's method offers a further insight into the converter's operation. From the instruction of the monodromy matrix (14), it is clear that the saltation matrices $\mathbf{S}_{\mathbf{1}}$ and $\mathbf{S}_{\mathbf{2}}$ play an important role in determining the eigenvalues of the monodromy matrix and hence the stability of the system. This leads to the possibility of altering the stability of the system by changing the saltation matrices $\mathbf{S}_{\mathbf{1}}$ and $\mathbf{S}_{\mathbf{2}}$. This idea has been previously employed in stabilizing discontinuous mechanical systems [6-8]. Equation (1) shows that the saltation matrix $\mathbf{S}$ depends on the two vector fields (which cannot change) and on the switching manifold $h$. Based on this insight, two alternative methods are proposed below to control the Neimark bifurcation in the Ćuk converter and maintain stability over a much wider range of bifurcation variables.

Changing the switching manifold by adding a small component to the control parameter $\mu$

This control method is based on altering the switching manifold by changing the normal vector $\mathbf{n}$. This can be achieved by adding a small component $a$ to the control parameter $\mu$. The new control law is given by:

$\left(x_{3}(d T)+x_{4}(d T)\right)=k-(\mu+a) x_{1}(d T) \pm \frac{\Delta i}{2}$

Hence, the normal vector will be:

$\mathbf{n}^{T}=\left[\begin{array}{llll}\mu+a & 0 & 1 & 1\end{array}\right]$

It is obvious that the eigenvalues of the monodromy matrix will be a function of $a$. The question is, how can we calculate the value of $a$ so that the stability characteristics remain the same over a larger range of $k$ ? For example, the value of $a$ that keeps the absolute value of the complex eigenvalues at 0.9998 can be calculated by solving the nonlinear equation.

$|0.9998 \times I-\mathbf{W}|=0$

The results of this algorithm for various values of $k$ are shown in Fig. 6. On the basis of the above observation, we have derived a look-up table which we have used to propose a supervising controller that adjusts the value of $a$ depending on the value of $k$. The response of this controller is shown in Fig. 7.

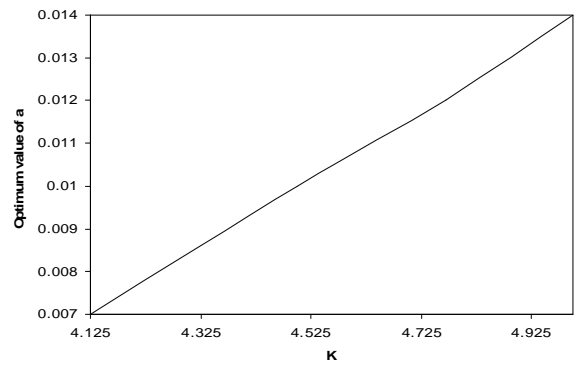

Fig. 6: Values of $a$ for stable operation. 


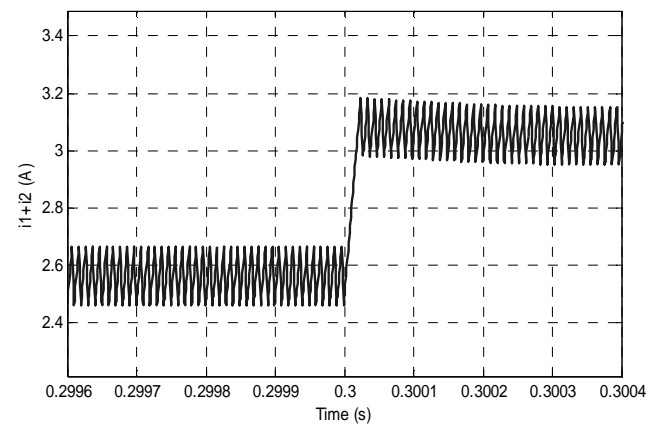

Fig. 7: Sum of inductor currents as $k$ increases from 3.375 to 4.125 .

Changing the switching manifold $h$ by adding a signal proportional to the output voltage $v_{2}$

This control method is based on changing the normal vector $\mathbf{n}$ by adding a signal proportional to the output voltage $v_{2}$ to the voltage feedback loop. This will change the second coordinate of the normal vector $\mathbf{n}$. The new control law is:

$\left(x_{3}(d T)+x_{4}(d T)\right)=k-\mu x_{1}(d T)+b x_{2}(d T) \pm \frac{\Delta i}{2}$

This will effectively alter the switching hypersurface $h$ to:

$h_{1}=\left(x_{3}(d T)+x_{4}(d T)\right)-k+\mu x_{1}(d T)-b x_{2}(d T)-\frac{\Delta i}{2}$

Hence, the normal vector will be:

$\mathbf{n}^{T}=\left[\begin{array}{llll}\mu & -b & 1 & 1\end{array}\right]$

Now we can design a controller to place the absolute value of the complex eigenvalues inside the unit circle corresponding to stable period one operation. This can be acheived by using the strategy explained in the previous controller. The results are shown in Fig. 8. The response of this controller is shown in Fig. 9.

\section{Conclusion}

The monodromy matrix, which defines the state transition matrix over one full cycle, has been used to study the stability analysis of the Cuk converter with a hysteresis current controller. Analysis of the autonomous system reveals that the system loses stability via Neimark Bifurcation. Two control methods based on the location of the Floqute multiplier of the system have been proposed, to force the system's eigenvalues within the unit circle and maintain stability for a wider range of values of the bifurcation parameters.

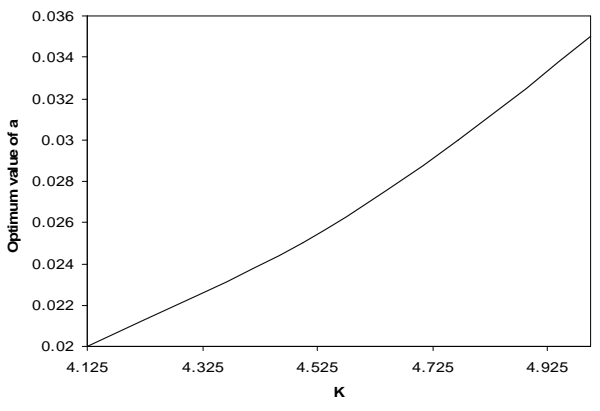

Fig. 8: Values of $b$ for stable operation.

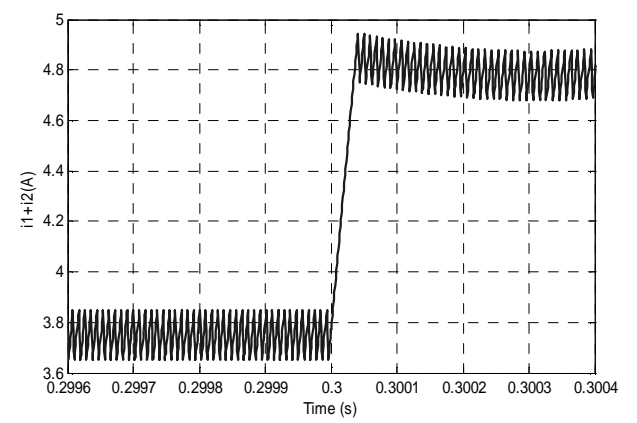

Fig. 9: Sum of inductor currents as $k$ increases from 3.375 to 4.125 .

\section{References}

[1] C. K. Tse, Y.M. Lai, and H.H.C. Iu, "Hopf bifurcation and chaos in a free-running current-controlled Cuk switching regulator," IEEE Transactions on circuits and system, vol. 47, pp. 448-457, 2000.

[2] D. Giaouris, S. Banerjee, B. Zahawi, and V. Pickert, "Stability Analysis of the Continuous Conduction Mode Buck Converter via Filippov's Method," IEEE transaction on circute and system-1, 2007.

[3] D. Giaouris, S. Banerjee, B. Zahawi, and V. Pickert, "Control of Fast Scale Bifurcations in Power-Factor Correction Converters," IEEE transaction on circute and system-1, vol. 54, pp. 805-809, 2007.

[4] A. F. Filippov, "Differential Equations with Discontinuous Righthand Sidees," Kluwer Academic Publishers, 1988.

[5] R. I. Leine and H. Nijmeijer, "Dynamics and Bifurcations of Non-Smooth Mechanical Systems," Springer, 2004.

[6] H. J. Dankowicz and P. T. Piiroinen, "Exploiting discontinuities for stabilization of recurrent motions," Dynamical Systems, vol. 17, pp. 317-342, 2002.

[7] H. J. Dankowicz and J. Jerrelind, "Control of neargrazing dynamics in impact oscillators," in Proc. Roy. Soc. London Ser. A, vol. 461, pp. 3365-3380, 2005.

[8] P. T. Piiroinen and H. J. Dankowicz, "Low cost control of repetitive gait in passive bipedal walkers," Int. J. Bifurc. Chaos, vol. 15, pp. 1959-1973, 2005. 